\title{
Effect of Wheat Germ Agglutinin (WGA) Binding to Red Blood Cell on Plasmodium falciparum Invasion
}

\author{
Naftal Kilenga* , Masahiko Kato, Sumie Manno, Akira Kaneko \# \\ and Yuichi Takakuwa
}

Department of Biochemistry and Department of International Affairs and Tropical Medicine\# , Tokyo Women's Medical University

8-1 Kawada-cho, Shinjuku-ku, Tokyo 162-8666, Japan

Red blood cell $(\mathrm{RBC})$ membranes are stable and deformable due to its mechanical properties maintained by the spectrin-based submembranous skeletal network. Therefore, RBC appears not to accept Plasmodium falciparum ( $\mathrm{Pf}$ ) invasion easily. However, Pf does invade RBC probably due to change in membrane mechanical properties at the area of Pf contact.

In the present study we examined whether increase in membrane rigidity may prevent Pf invasion into $\mathrm{RBC}$. Wheat germ agglutinin (WGA) was utilized to increase membrane rigidity and double fluorescence assay with a flow cytometer was established; the biotin-WGA-treated RBC (B-WGA-treated RBC) was mixed with Pf-infected RBC followed by staining with streptavidin-FITC and propidium iodide (PI) to detect BWGA-treated RBC and Pf-infected RBC, respectively.

Having confirmed that membrane rigidity of B-WGA-treated RBC increased significantly using an ektacytometer, we could show that these cells were not infected by Pf using double fluorescence assay. The target molecule of B-WGA was also identified to be band 3 by pull-down assay. These results enabled us to suggest that B-WGA binding to band 3 molecule may induce conformational change in band 3-ankyrin-spectrin, sequentially, resulting in increase in membrane rigidity and thus prevention of Pf invasion into RBC.

Key words : Plasmodium falciparum / red blood cell $/$ membrane rigidity / wheat germ agglutinin $/$ band 3

\section{Introduction}

Malaria is the most serious disease of human red blood cell ( $\mathrm{RBC}$ ) caused by Protozoan parasite of the genus Plasmodium in that P. falciparum (Pf) is responsible for the most severe form of malaria ${ }^{1}$. About 300-400 million people are infected

* Corresponding Author

Tel : +255-22-2-151120

E-mail : per year worldwide with an average of 6,000 (including 3,000 children) deaths per day ${ }^{1,2)}$. Sporozoites invade the liver cells and multiplying to thousands followed with release of merozoites. After successful internalization of the merozoite into $\mathrm{RBC}$, asexual division starts and the parasite develop from ring to mature stage and eventually bursts to release merozoite that can reinvade $\mathrm{RBC}$ (Fig. 1).Morbidity and mortality occur during the intra-RBC stage of the life cycle of the parasite, and 


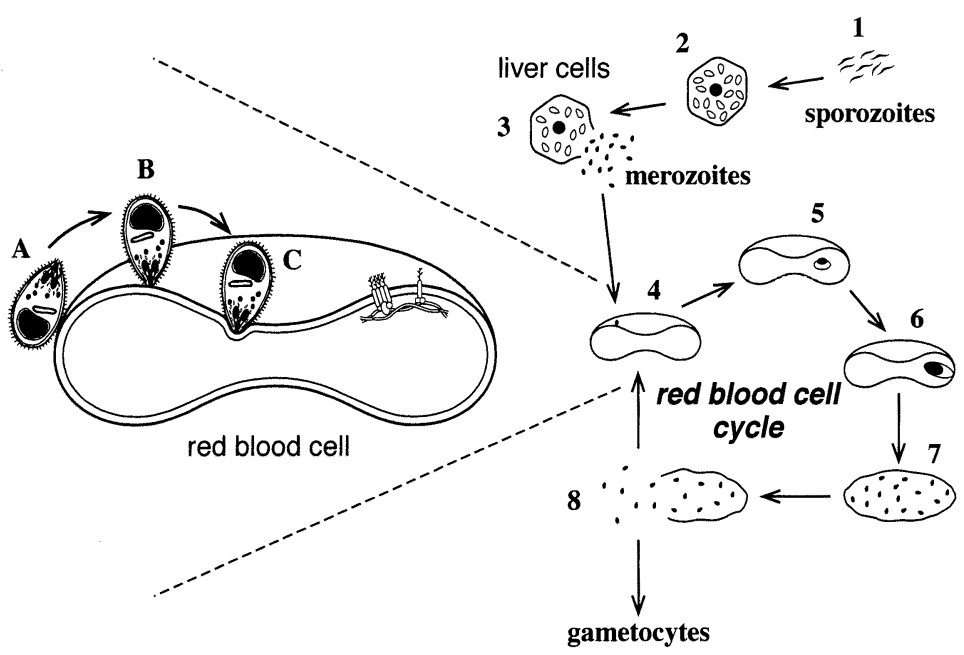

Fig. 1 P.falciparum (Pf) hepatic and red blood cell (RBC) cycle.

Sporozoites are inoculated to human blood circulation by mosquito (1). The sporozoites invade the liver cells and multiply to thousands (2). Mature schizonts rupture and release merozoites (3), the merozoites invade into $\mathrm{RBC}$, within $\mathrm{RBC}$, the parasites multiply by asexual division, Pf develop through ring, trophozoite and mature schizont stage, respectively (4-7). The mature schizont bursts to release 8-24 merozoites (8), which reinvade new RBC or merozoites may differentiate into sexual RBC stages (gametocytes).

it is this stage that most scientists are investigating for drug and vaccine developments.

$\mathrm{RBC}$ membrane is stable and reasonably rigid due to its mechanical properties maintained by the spectrin-based submembranous skeletal network. Therefore, RBC appears not to accept Pf invasion easily. However, Pf does invade RBC probably due to change in membrane mechanical properties at the area of Pf contact $^{3}$. It is thus reasonable to assume that upon Pf contact with RBC, membrane rigidity may locally decrease due to signal transduction through conformational changes of transmembrane protein-anchor protein-membrane skeletal proteins, such as spectrin. In the present study we examined whether increase in membrane rigidity prevent Pf invasion into $\mathrm{RBC}$. For this purpose wheat germ agglutinin (WGA) was utilized since this lectin has been shown to increase rigidity of $\mathrm{RBC}$ membranes ${ }^{4}$. In order to quantify para- sitemia (number of Pf-infected RBC) we used our newly developed double fluorescence assay with a flow cytometer. The biotin-WGA-treated RBC (BWGA-treated RBC) was mixed with Pf-infected $\mathrm{RBC}$ followed by staining with streptoavidin-FITC to detect B-WGA-treated RBC. The propidium iodide (PI) which specifically binds to DNA was simultaneously used to detect DNA in Pf-infected RBC.

Having confirmed that membrane rigidity of BWGA-treated RBC increased significantly using an ektacytometer, we could show that these cells were not infected by Pf using double fluorescence assay as described above. The target molecule of B-WGA was also identified to be band 3 by pulldown assay. These results enabled us to suggest that B-WGA binding to band 3 molecule may induce conformational change in band 3-ankyrinspectrin, sequentially, resulting in increase in 
membrane rigidity and prevention of Pf invasion.

\section{Materials and Methods}

\section{Materials}

Human venous blood was obtained from healthy volunteers after obtaining informed consent. Blood was mixed with anticoagulant-Citrate Phosphate Dextrose $(\mathrm{CPD})$ and stored at $4{ }^{\circ} \mathrm{C}$. The blood was used within three days after collection. For Pf culture, whole blood was mixed with anticoagulantAcid Citrate Dextrose $(\mathrm{ACD})$ and left at $4^{\circ} \mathrm{C}$ for at least 5 days or fresh blood was used after removal of leukocytes using Terumo kit III-RC (TERUMO, Japan). For preparation of nonimmune serum, blood from group $\mathrm{O}(+)$ donor was left to clot and the serum was collected and inactivated at $56^{\circ} \mathrm{C}$ for $30 \mathrm{~min}$. Biotin-wheat germ agglutinin (B-WGA), Streptavidin-FITC were from VECTOR Laboratories (Canada) and Wako (Tokyo, Japan) respectively. Polyvinylpiridoline (PVP) K 90 and Propidium iodide (PI) were obtained from Nacalai tesque Inc. (Kyoto, Japan). Monoclonal anti-glycophorin A (GPA) and glycophosin C (GPC) antibodies were obtained from Sigma-Aldrich (USA). Streptavidin Sepharose, Anti-Rabbit Immunoglobulins conjugated to Horse Radish Peroxidase were purchased from Amersham Pharmacia Biotech. (UK) and (DAKO A/S, Denmark), respectively. Polyclonal anti-band 3 antibody was produced in our laboratory. RPMI 1640 Medium powder with L-glutamine, $7.5 \%(\mathrm{v} / \mathrm{w}) \mathrm{NaHCO}_{3}$, and Hepes were ordered from GIBCO (USA). Chloroquine-sensitive strain P. falciparum F32 was obtained from Karolinska Institute (Sweden).

\section{Measurement of RBC membrane rigidity}

Twenty $\mu 1$ of washed RBCs were mixed with 4 $\mathrm{ml}$ of $3.5 \%$ PVP in PBS containing 0, 0.5, 1.0, 2.5 and $5.0 \mu \mathrm{g} / \mathrm{ml}$ of B-WGA, followed by incubation at room temperature with gentle mixing. Membrane rigidity was measured by an ektacytometer as described previously ${ }^{5}$. Briefly, RBCs were subjected to linearly increasing shear stress up to 150 dynes $/ \mathrm{cm}^{2}$, and change in laser diffraction patterns from a circle to an ellipse was recorded as a deformability index (DI ${ }^{6}$. . DI provides a measure of ellipticity of deforming RBC (namely spectrin extensibility) in the rotational flow field ${ }^{5}$. DI was plotted as a function of applied shear stress. DI increase curve indicates RBC membrane rigidity and thus the slower increase reflects higher rigidity.

\section{Treatment of RBC with B-WGA for in vitro Pf invasion assay}

B-WGA $(0$ and $5 \mu \mathrm{g} / \mathrm{ml})$ in PBS were mixed with human $\mathrm{RBC}$ of group $\mathrm{O}(+)$ followed by incubation at room temperature for 20 min with gentle mixing. The mixture was then washed three times in PBS to remove unbound B-WGA. At these concentrations neither cell aggregation nor haemolysis was observed. The RBC pellets with or without B-WGA were used for in vitro Pf invasion assay. Blood of group $\mathrm{O}(+)$ was used generally for Pf culture because it is compatible with serum or plasma of all other blood groups ${ }^{7}$.

\section{Preparation of Pf-infected RBC for in vitro Pf invasion assay}

Pf was grown in continuous culture ${ }^{8)}$ with RPMI 1640 medium supplemented with $10 \%$ heat-inactivated $\mathrm{O}(+)$ non-immune human serum, $25 \mathrm{mM}$ HEPES, $25 \mathrm{mg} / 1$ gentamycin sulfate and $24 \mathrm{mM}$ $\mathrm{NaHCO}_{3}$ and a $2 \%$ suspension of human $\mathrm{RBC}$ of group $\mathrm{O}(+)$ at $37^{\circ} \mathrm{C}$ under gas conditions of $7.5 \%$ $\mathrm{O}_{2}, 5 \% \mathrm{CO}_{2}$ and $87.5 \% \mathrm{~N}_{2}$.

Stages of Pf were synchronized with sorbitol ${ }^{9)}$ followed by continuous culture to obtain mature 
stage parasites such as late trophozoite and schizonts. Sorbitol was used to eliminate chemical or temperature stress that may affect the invasive stage of Pf. RBCs containing sorbitol-synchronized mature parasites, hereafter referred to as an inoculum, was used throughout our experiments. Percentage of parasitemia was calculated to be about $10 \%$ by counting the number of infected RBC in 1,000 RBC with Giemsa staining under light microscopy. It should be pointed out that the inoculum contained not only mature Pf-infected RBC (about 10\%) but also a significant number of Pf-uninfected intact RBC (about 90\%).

\section{In vitro $P f$ invasion assay}

An inoculum (containing Pf-infected and -uninfected RBCs) was mixed with B-WGA-treated or untreated RBCs in complete medium RPMI 1640 at a ratio of 1 to 18 . One $\mathrm{ml}$ of the mixture was dispensed to each of the micro well culture plate in triplicate followed by incubation at $37^{\circ} \mathrm{C}$ with $7.5 \%$ $\mathrm{O}_{2}, 5 \% \mathrm{CO}_{2}$ and $87.5 \% \mathrm{~N}_{2}$ for $48 \mathrm{hr}$.

For flow cytometric analysis, double fluorescence of streptavidin-FITC and PI were used to detect B-WGA-treated RBC and DNA of Pf-infected $R B C$, respectively. $R B C$ were harvested, washed and re-suspended in PBS. Streptavidin-FITC was added and incubated at room temperature for 30 min in the dark. After washing, RBC in the pellet was fixed and permeabilized as described ${ }^{10)}$ with slight modification. Briefly, RBC was suspended in $0.025 \%$ glutaraldehyde in PBS to a haematocrit of $2 \%$ and incubated at room temperature for $20 \mathrm{~min}$. After washing, RBC was permiabilized with $1 \%$ (v/w) saponin in PBS containing 1\% BSA, followed by incubation at room temperature for 15 min. After washing, $10 \mu \mathrm{g} / \mathrm{ml}$ of PI was added and left at room temperature for $20 \mathrm{~min}$ in the dark.
Resolution was checked for each sample by using fluorescence microscope (Eclipse E600, Nikon, Japan) before proceeding to flow cytometric analysis. Sample was applied and quantified by the flow cytometer (EPICS-XL,Beckman Coulter Co.).

\section{Pull-down assay}

$\mathrm{RBCs}(100 \mu \mathrm{l})$ were suspended in $4 \mathrm{ml}$ PBS containing B-WGA $(4 \mu \mathrm{g} / \mathrm{ml})$ and incubated for $30 \mathrm{~min}$ at room temperature. Cells were collected, washed with PBS, and suspended in PBS containing $1 \%(\mathrm{v} / \mathrm{v})$ Triton X-100. After incubation on ice for $30 \mathrm{~min}$, the suspension was centrifuged at 6,000 $\times \mathrm{g}$ for $10 \mathrm{~min}$ at $4^{\circ} \mathrm{C}$. The supernatant (TX-sup) was collected and the pellets re-suspended in 0.1 mM EDTA (pH8.3) followed by incubation at $37^{\circ} \mathrm{C}$ for $1 \mathrm{hr}$. Following centrifugation $(100,000 \times$ g, $15 \mathrm{~min}$ ) collected supernatant was diluted with PBS (EDTA-sup). Streptavidin-Sepharose was added to these supernatants (TX-sup and EDTAsup) followed by incubation at $4^{\circ} \mathrm{C}$ for $2 \mathrm{hr}$ or over night with gentle mixing. The Sepharose beads were collected by sedimentation, washed by PBS, and re-suspended in the washing buffer $(50 \mathrm{mM}$ Tris- $\mathrm{HCl}$ (pH 7.5), $150 \mathrm{mM} \mathrm{NaCl}, 1 \%$ (w/v) Nonidet P-40, 0.1\% (w/v) SDS, and 0.5\% (w/v) sodium deoxycholate). After incubation at room temperature for $10 \mathrm{~min}$ with gentle mixing the beads were collected and further incubated in 10 $\mathrm{mM}$ phosphate buffer $(\mathrm{pH} 7.4)$ containing $1 \mathrm{M}$ $\mathrm{NaCl}$ for 10 min. Pulled-down proteins were denatured in SDS-sample buffer $(125 \mathrm{mM}$ Tris-HCl (pH6.8), 4.6\% (w/v) SDS, 20\% (v/v) glycerol, $10 \%(\mathrm{v} / \mathrm{v}) 2$-mercaptoethanol, and $20 \mu \mathrm{g} / \mathrm{ml}$ Bromophenol Blue) by heating at $100^{\circ} \mathrm{C}$ for $5 \mathrm{~min}$. The samples were then analyzed by $9 \%$ SDS-PAGE followed by silver stain and immunoblotting ${ }^{11)}$. 


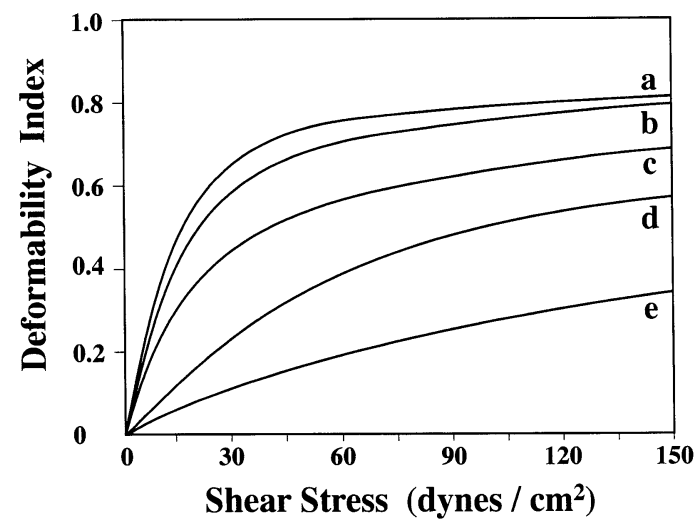

Fig. 2 Measurement of RBC membrane rigidity using an ektacytometer.

DI of the control RBC (line a) and B-WGA-treated RBC (lines b-e) were monitored with increasing shear stress up to $150 \mathrm{dyn} / \mathrm{cm}^{2}$. DI for the control RBC increased rapidly to reach DImax of 0.8 (line a) whereas B-WGA-treated RBC showed a slower rate of DI increase dependent on B-WGA concentrations of 0.5 (line b), 1.0 (line c), 2.5 (line d) and 5.0 (line e) $\mathrm{mg} / \mathrm{ml}$, respectively.

\section{Results}

\section{Increase in $\mathrm{RBC}$ membrane rigidity by $\mathrm{B}$ -} WGA

Membrane rigidity of B-WGA-treated RBC were measured by an ektacytometer (Fig. 2). When the control RBCs were subjected to linearly increasing applied shear stress up to $150 \mathrm{dyn} / \mathrm{cm}^{2}$, the deformability index (DI) increased, reaching a maximum DI level (DImax) of 0.8 (line a). BWGA-treated RBCs showed a slow DI increase curve, indicating that the membrane rigidity was increased by B-WGA treatment. This effect was enhanced with increase in B-WGA concentrations up to $5 \mu \mathrm{g} / \mathrm{ml}$ (lines b-e). Since shape of B-WGAtreated RBC still showed normal biconcave disc form (data not shown), DI increase corresponds mainly to the extensibility of spectrin. If that is the case, B-WGA binding on the RBC surface may induce decrease in spectrin extensibility in the submembranous membrane skeleton through some transmembrane signal transduction. It should be noted that neither cell aggregation nor haemolysis was observed at B-WGA concentrations up to $5 \mu$ $\mathrm{g} / \mathrm{ml}$.

\section{Flow cytometric measurement of Pf infection}

We examined whether Pf can invade into BWGA-treated RBC with extremely rigid membranes by a flow cytometric measurement as described in Materials and Methods. Two fluorescent dyes such as streptavidin-FITC and PI were used to detect B-WGA-treated RBC and Pf-infected $\mathrm{RBC}$, respectively. Simultaneous detection using FITC and PI enabled us to observe separately 4 populations in each window 1 to 4 (see figure legend for Fig. 3A).

Using Pf-uninfected RBC with or without B-WGA treatment, we firstly demonstrated that these two RBC populations could be observed separately by their differences in FITC fluorescence intensity. As shown in Fig. 3B, following mixing equal numbers of RBC with low FITC intensity and B-WGA-treated $\mathrm{RBC}$ with high FITC intensity, almost equal distribution was observed in windows 1 and 2 .

Using WGA-untreated RBC, we then made sure that Pf-infected RBC were detected separately from Pf-uninfected RBC by their differences in PI fluorescence intensity. As shown in Fig. 3C, following $48 \mathrm{hrs}$ incubation of the inoculum (containing 10\% Pf-infected and $90 \%$-uninfected $\mathrm{RBC}$ ) with $\mathrm{RBC}$ at a ratio of $1: 18$, the flow cytometric analysis of this sample showed that $4.4 \%$ of Pf-newly infected RBC with high PI intensity was observed in window 3 and $93.4 \%$ of Pf-uninfected RBC with low PI intensity in window 1 . It should be noted that original Pfinfected RBC (10\%) were ruptured during incuba- 
A
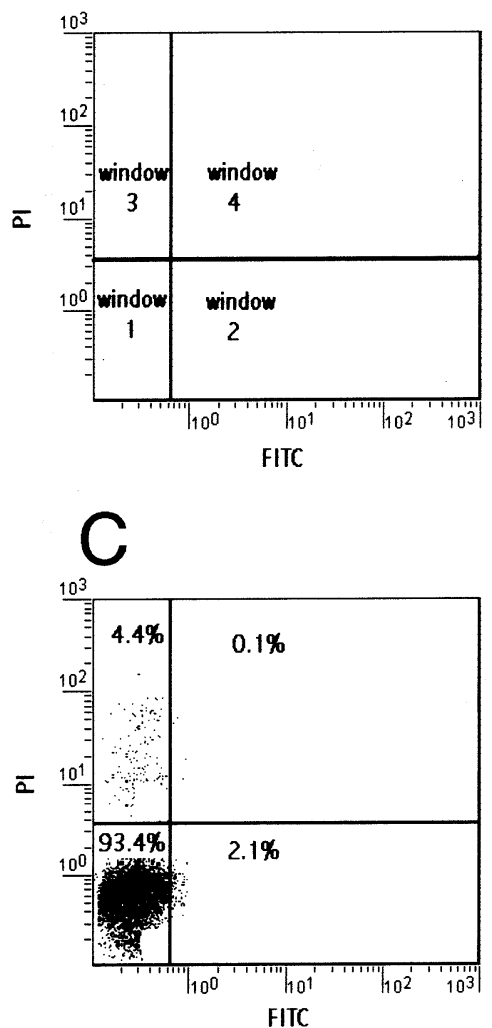

B
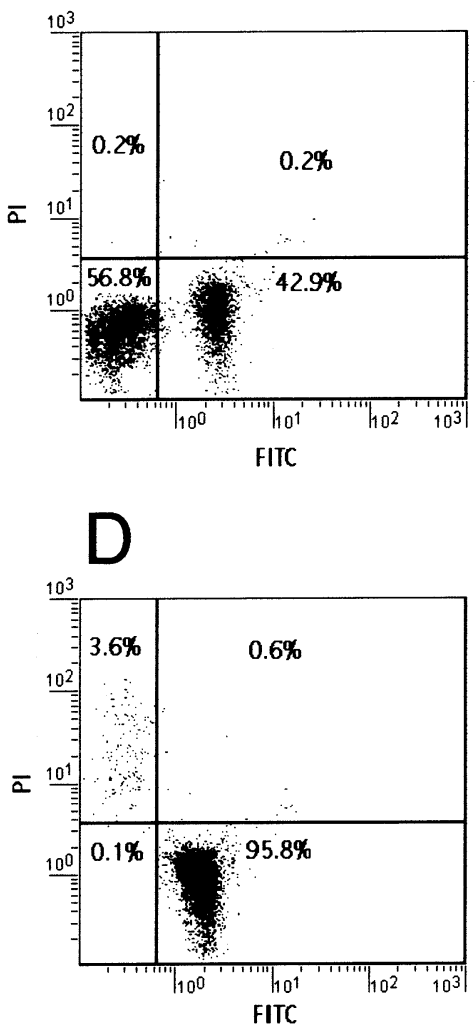

Fig. 3 Flow cytometric analysis of Pf infection into B-WGA treated RBC.

RBCs were analyzed by a flow cytometer (EPIC-XL, Beckman Coulter Co.) as described in Materials and Method. Analysis windows were set up for FITC on the X-axis and PI on the Y-axis. Fig. 3A will show definitions for each window; Window 1 corresponds to the control RBC that is B-WGA-untreated and Pf-uninfected RBC. Window 2 represents B-WGAtreated but Pf-uninfected RBC. In window 3 there are B-WGA-untreated but Pf-infected RBC. Window 4 represents WGA-treated and Pf-infected RBC that is stained with both FITC and PI. Fig. 3B shows distribution of the mixture of B-WGA-treated and -untreated $\mathrm{RBC}$ at a ratio of 1 to 1 . Fig. $3 \mathrm{C}$ represents distrubution of the mixture of Pf-infected and uninfected $\mathrm{RBC}$ at a ratio of 1 to 18 after $48 \mathrm{hrs}$ incubation. Fig. 3D shows distribution of the mixture of Pf-infected RBC and B-WGA-treated RBC at a ratio of 1 to 18 after $48 \mathrm{hrs}$ incubation. It should be noted that Pf-infected RBC sample （inoculum） contains $10 \% \mathrm{Pf}$ infected $\mathrm{RBC}$ and $90 \%$ Pf-uninfected RBC. The number of Pf even after RBC rupture is much less than the total number of RBC in Figs. 3C and 3D.

tion, releasing an average of $10 \mathrm{Pf}$ per RBC. These Pf infected both RBCs which were contained in the inoculum (containing 90\% Pf-uninfected RBC) and newly added RBC that is 18 times the number of the inoculum.

Having established the flow cytometric analysis 




B. anti-band 3
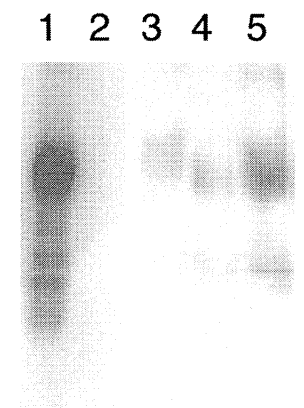

D. anti-GPC

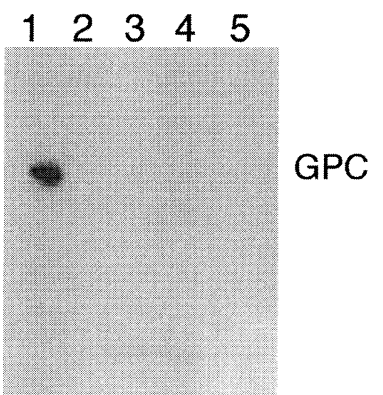

Fig. 4 Identification of the B-WGAbinding protein on $\mathrm{RBC}$.

B-WGA was pulled-down from TX-sup and EDTA-sup by streptavidin-Sepharose as described for Material and Methods. Proteins pulleddown with B-WGA from TXsup (lanes 2 and 3 ) and EDTA-sup (lanes 4 and 5) were analyzed by SDS-PAGE (9\%). The supernatants were prepared from B-WGAuntreated (lanes 2 and 4) or -treated (lanes 3 and 5) RBC. Panel A: silver stain. Panel B-D : immunoblotting using anti-band $3(\mathrm{~B})$, antiGPA (C), and anti-GPC (D) antibodies. Ghost was used as standard for RBC membrane proteins (lane 1 ). technique we examined whether Pf invaded into BWGA-treated RBC. As shown in Fig. 3D, following 48 hrs incubation of Pf-infected RBC sample with B-WGA-treated RBC at a ratio of $1: 18$, the flow cytometric analysis of this sample showed that $3.6 \%$ of Pf-newly infected RBC with high PI intensity was observed in window 3 and $95.8 \%$ of Pf-uninfected RBC with high FITC intensity was seen in window 2. The fact that no $\mathrm{RBC}$ was observed in window 4 indicates that Pf did not invade into BWGA-treated RBC. It should be noted that $3.6 \%$ of Pf-newly infected RBC in window 3 were derived from about $90 \%$ of Pf-uninfected RBC which were contained in the inoculum.

\section{Identification of the B-WGA-binding protein on RBC}

To identify B-WGA-binding proteins on $\mathrm{RBC}$ sur- face, we used pull-down assay with streptavidinSepharose as described in Materials and Methods. Since membrane skeletal proteins were not easily solubilized by $1 \%(\mathrm{v} / \mathrm{v})$ Triton-X100 (due to tight associations to each other), two-step solubilization was performed as described for Materials and Methods. Solubilized B-WGA was pulled-down by streptavidin-Sepharose and proteins simultaneously sedimented were analyzed by SDS-PAGE and immunoblotting. As shown in Fig. 4A, band 3 was detected in TX-sup (lane 3) as well as EDTA-sup (lane 5). Presence of band 3 was confirmed by immunoblotting with an anti-band 3 antibody (Fig. 4B, lanes 3 and 5). These results indicate that under the present experimental conditions BWGA appears to bind to sugar chains of band 3 protein. We then examined whether GPA and GPC were also recognized by B-WGA since these gly- 
cophorins with several sugar chains are considered to be a possible binding molecule of WGA. GPA was detected as a dimer and a monomer on RBC ghost (Fig. 4C, lane 1) while GPA was not detected in pulled-down fraction in TX-sup and EDTA-sup (Fig. 4C, lanes 3 and 5). GPC present as a monomer on $\mathrm{RBC}$ ghost (Fig. 4D, lane 1) was not detected in both pulled-down fractions (Fig. 4D, lanes 3 and 5). These results indicate that band 3 , but not GPA and GPC, is at least one of the B-WGA-binding protein on $\mathrm{RBC}$.

\section{Discussion}

In the present study we could show that increase in membrane rigidity prevented invasion of $\mathrm{Pf}$ into RBC. B-WGA was used to increase in membrane rigidity. To quantitate parasitemia a flow cytometer was utilized for double fluorescence assay with streptavidin-FITC and PI to detect B-WGA-treated $\mathrm{RBC}$ and Pf-infected RBC, respectively.

We first confirmed that B-WGA (as well as WGA) increased $\mathrm{RBC}$ membrane rigidity using an ektacytometer. This is consistent with the previous report showing WGA-induced increase in $\mathrm{RBC}$ membrane rigidity ${ }^{4}$. Since the shape of B-WGAtreated RBC still showed normal biconcave disc form, the observed increase in rigidity may not be derived from loss of effective surface area ${ }^{4)}$. Although WGA at high concentrations may aggregate RBCs, neither cell aggregation nor haemolysis was observed at B-WGA concentrations we used in the present study. These results enabled us to use B-WGA-treated RBC as RBC with significantly increase in membrane rigidity.

Membrane rigidity is maintained by the spectrinbased membrane skeleton which underlies RBC membranes. Especially increase and decrease in extensibility of spectrin decrease and increase in membrane rigidity, respectively. The membrane skeleton interacts with transmembrane proteins such as band 3 and GPC through anchor proteins of ankyrin and protein 4.1 , respectively ${ }^{12)}$. In the present experiments, band 3 was identified as the only target for B-WGA using a pull-down assay (Fig. 4), indicating that the signal of B-WGA binding to band 3 reached spectrin molecule through conformational change of band 3-ankyrin-spectrin sequentially and the extensibility of spectrin might decrease with an increase in rigidity.

Having confirmed the effect of B-WGA we examined whether an increase in $\mathrm{RBC}$ membrane rigidity may prevent Pf invasion into $\mathrm{RBC}$ using B-WGAtreated RBC. We established a flow cytometric analysis with double fluorescences, streptoavidinFITC and PI, to quantitatively measure parasitemia of B-WGA-treated and -untreated RBC. With this technique we have been able to separate $\mathrm{RBC}$ into four distinct populations with combinations of FITC (+/-) and PI (+/-) (Fig. 3). The results clearly showed that there was no detectable double positive RBC (Fig. 3D), indicating that B-WGA treatment prevented Pf invasion into RBC.

Mechanism for WGA-induced prevention of Pf invasion is most likely due to increase in $\mathrm{RBC}$ membrane rigidity. $\mathrm{RBC}$ membranes with increased rigidity must not be easily invaginated following Pf contact. However, we cannot completely exclude the (possible) competition for $\mathrm{RBC}$ receptor between WGA and Pf-derived factors, such as $\mathrm{MSP} 1^{13)}$ and EBA-175 ${ }^{14,15)}$ that are necessary for Pf invasion. Since MSP1 has been shown to bind band 3, B-WGA may compete with MSP1 for band 3 on the RBC surface. Furthermore, GPA has been reported as a $\mathrm{RBC}$ receptor for EBA-175 ${ }^{14,16)}$ and target for $\mathrm{WGA}^{4,17}$. Therefore, one might suggest that WGA competes with EBA175 for GPA on the RBC surface, resulting in prevention of Pf invasion. However, in our hands band 3 (not GPA) was only the target of B-WGA 
(Fig. 4). It is, therefore, unlikely for B-WGA to interrupt binding of EBA-175 to GPA.

In the present experiments the increase in rigidity by WGA is equally distributed through the entire RBC membranes and as such Pf poorly invaded. Although WGA cannot be used in vivo as anti-parasite invasion drug, some harmless factor affecting membrane mechanical properties may be available as a drug for prevention of Pf invasion in future. Moreover, present results enable us to assume that during initial stage of Pf invasion the $\mathrm{RBC}$ membrane rigidity is locally decreased, allowing invagination upon Pf contact. Identification of Pf-derived factors which induces decrease in membrane rigidity is required to understand precisely the molecular mechanism for Pf invasion into RBC.

Abbreviations : Pf, Plasmodium falciparum; RBC, red blood cell; B-WGA, biotin-wheat germ agglutinin; GPA, glycophorin A; GPC, glycophorin C; FITC, fluoroscein isothiocyanate

\section{Acknowledgements}

The authors would like to appreciate Prof. Kobayakawa T. for useful discussion and Dr. Takahashi N., Ms. Miyazawa M., and Dr. Bwijo A.B. for technical advise regarding Pf culture in the Department of International Affairs and Tropical medicine. We are grateful to Dr. Yagi J., Dr. Kato H., and Prof. Uchiyama T. in the Department of Microbiology and Immunology for technical advice regarding use of the flow cytometry. Ms. Ito $\mathrm{M}$. in our laboratory is appreciated for measurement of membrane regidity by an ektacytometer. We also appreciate Dr. Mohandas N. at the New York Blood Center for useful suggestions. This work was supported in part by Grant-in-Aid for Scientific
Research 15019101 from the Ministry of Education and Science of Japan and Japan International Cooperation Agency (JICA) for Kilenga N.

\section{References}

1) Breman JG, Egan A, Keusch GT : Am.J.Trop.Med. Hyg., 64 iv-vii (2001)

2) Seattle Biomedical Research Institute. Malaria statistics News. (2003)

3) Aikawa M, Miller LH, Johnson J, Rabbege J : J. Cell Biol., 77 72-82 (1978)

4) Chasis JA, Schrier S : Blood, 70 2562-2568 (1989)

5) Mohandas N, Clark MR, Jacobs MS, Shohet SB : J. Clin. Invest., 66 567-573 (1980)

6) An XL, Takakuwa $Y$, Nunomura $W$, Manno $S$, Mohandas N : J. Biol.Chem., 271 33187-33191 (1996)

7) Read M, Hyde JE : "Protocols in Molecular Parasitology”, Jensen JB ed,Vol. 21. Humana Press. NJ (1993)

8) Trager W, Jensen JB : Science, 193 673-675 (1976)

9) Lambrose C, Vandeberg JP : J. Parasitol, 65 418-420 (1979)

10) Pattanapanyasat K, Udomsangpetch $R$, Webster HK : Cytometry, 14 449-454 (1993)

11) Nagao K, Takakuwa $Y$, Manno S, Suzuki H : Membrane, 20 287-295 (1995)

12) Takakuwa Y:Int J. Hematol., 72 298-309 (2000)

13) Goel VK, Li X, Chen H, Liu S-C, Chishti AH, Oh SS : Proc. Natl. Acad. Sci. U.S.A., 100 5164-5169 (2003)

14) Mayer DCG, Kaneko O, HudsonTaylor D, Reid M, Miller LH : Proc. Natl. Acad. Sci. U.S.A., 98 52225227 (2001)

15) Gilberger T, Thompson JK, Triglia T, Good RT, Duraisingh MT, Cowman AF : J. Biol. Chem., 278 14480-14486 (2003)

16) Orlandi PA, Klotz FW, Haynes JD : J. Cell Biol., 116 901-909 (1992)

17) Chasis AJ, Reid ME, Jensen RH, Mohandas $\mathrm{N}: J$. Cell Biol., 107 1351-1357 (1988)

(Received 12 December 2003;

Accepted 16 December 2003) 